\title{
Fuzzy approximation of the functions minimum and maximum
}

\author{
B.Sládek \\ The Gerstner Laboratory, Czech Technical University Prague \\ Technicka 2, CZ166 27 Praha 6, Czech republic. \\ e-mail: sladek@control.felk.cvut.cz
}

\begin{abstract}
This paper deals with the approximation of the functions minimum and maximum. Both standard and fuzzy algorithms are discussed and practical applications in trajectory planning and drive control are presented.
\end{abstract}

\section{Keywords \\ Fuzzy control, trajectory planning}

\section{INTRODUCTION}

Minimum and maximum belong to the most useful nonlinear functions in the control theory. The linear control theory is an efficient tool for control of dynamic systems whose states and control actions operate safely within their limits. If safety margins are not wide enough and limits on system states and control actions have to be taken into accounts, nonlinear control systems have to be used. Functions minimum and maximum are frequently used in nonlinear feedback controllers but their disadvantage is that they are not smooth. Therefore, there are several approximative methods, which are used if smooth output is desired.

Some criteria for the choice of the best method are:

1. Tuning knobs used to modify the shape of the transient area.

2. Symmetry.

3. Computational demand.

4. Degree of continuity.

5. Overshoot. 


\section{APPROXIMATION FORMULAS}

\subsection{Frequently used symbols}

$y\left(x, x_{\mathrm{m}}\right)$ the resulting function approximating $\min \left(x, x_{\mathrm{m}}\right)$

$\mu_{\mathrm{Xx}}($ ) membership degree of the fuzzy set $X X$.

$f_{\mathrm{XX}}(\mathrm{)}$ conclusion function corresponding to the label $X X$

$k_{\mathrm{H},} k_{\mathrm{L},} k_{\mathrm{S}}$ width of the high(low, symmetric) portion of the transient area

\subsection{Exact minimum}

$$
y\left(x, x_{m}\right)=\left\{\begin{array}{cc}
x & \text { if } x<x_{m} \\
x_{m} & \text { else }
\end{array} .\right.
$$

Properties of the function $\min \left(x, x_{\mathrm{m}}\right)$ are shown in the Figure 1. Dotted lines in the contour plot separate the transient area.
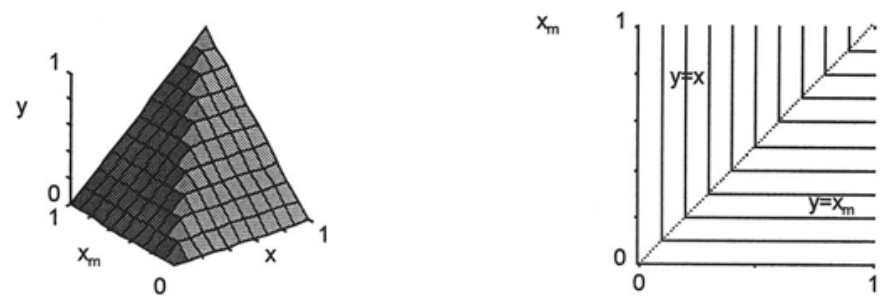

Figure 1. Exact function $\min \left(x, x_{\mathrm{m}}\right)$.

\subsection{N-th power}

This is the most frequent way of smooth approximation of $\min \left(x, x_{\mathrm{m}}\right)$ :

$$
f_{Z}\left(x, x_{m}\right)=\sqrt[n]{x^{n}+x_{m}^{n}} \text {. }
$$

The width of the transient area depends on the order $n$. Realistic width of the transient area is limited by numeric problems caused by calculation of high powers of $x$.
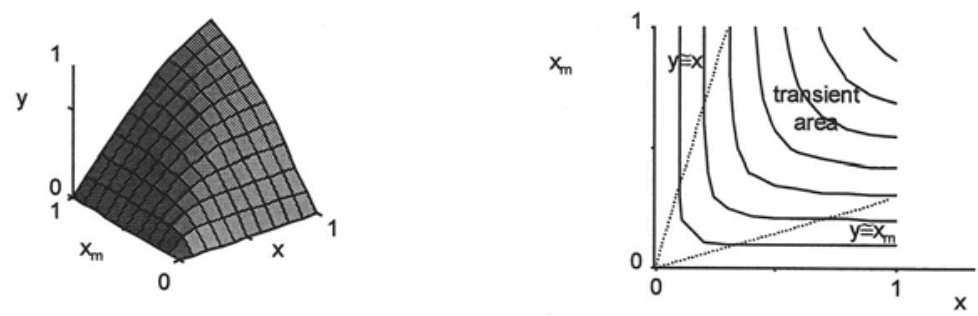

Figure 2. Polynomial approximation of $\min \left(x, x_{\mathrm{m}}\right)$ using $x^{\mathrm{n}}$. 


\subsection{Fuzzy approximation with 2 rules}

It is easy to describe the function $\min \left(x, x_{\mathrm{m}}\right)$ by a Takagi-Sugeno fuzzy model. The crisp function $\min \left(x, x_{\mathrm{m}}\right)$ is equivalent to a fuzzy system with 2 rules, rectangular membership functions and linear conclusion functions $x$ and $x_{\mathrm{m}}$.

Unfortunately, simple change from rectangular to trapezoidal membership functions gives poor results: the resulting function $y\left(x, x_{\mathrm{m}}\right)$ is non-smooth and has an overshoot.

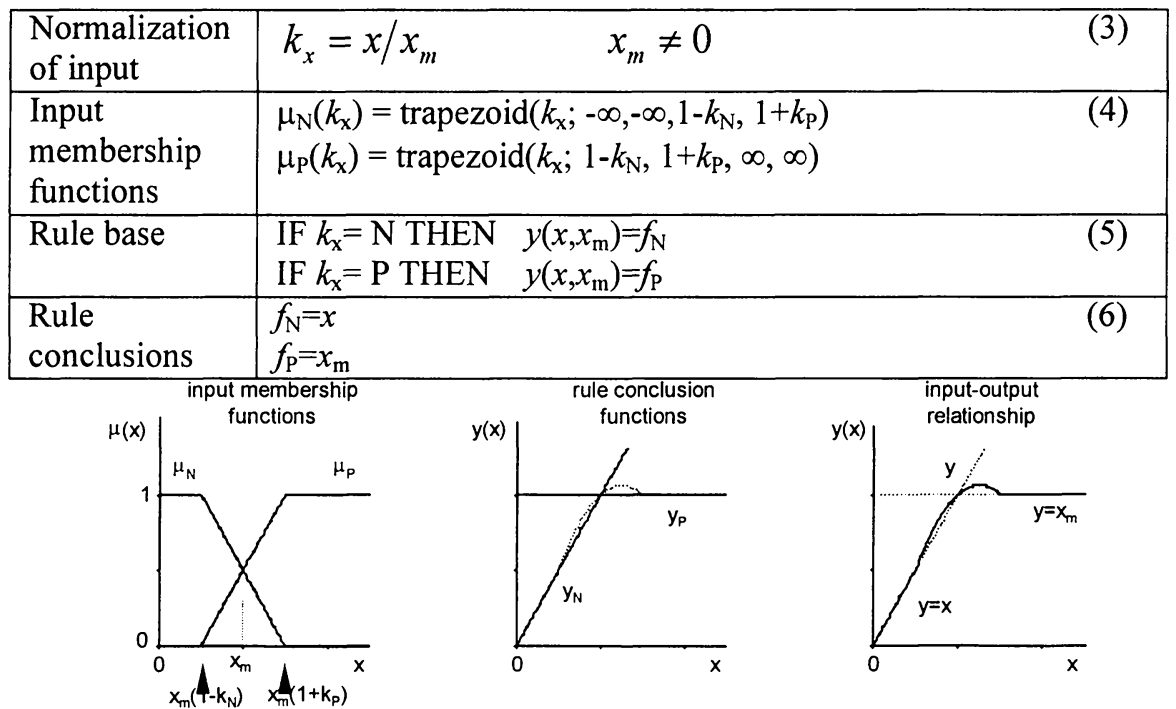

Figure 3. Fuzzy system with 2 rules: input membership functions, conclusion functions and input-output relationship.
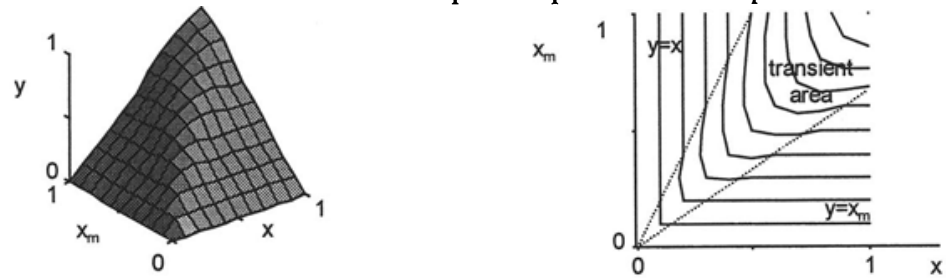

Figure 4. Fuzzy approximation of $\min \left(x, x_{\mathrm{m}}\right)$ with 2 rules.

\subsection{Fuzzy approximation with 4 rules}

To obtain a practical solution, 4 rule system is a minimum.

\begin{tabular}{|l|l|}
\hline Input & $\mu_{\mathrm{LN}}\left(k_{\mathrm{x}}\right)=\operatorname{trapezoid}\left(k_{\mathrm{x}} ;-\infty,-\infty, 1-k_{\mathrm{N}}, 1\right)$ \\
membership & $\mu_{\mathrm{SN}}\left(k_{\mathrm{x}}\right)=\operatorname{trapezoid}\left(k_{\mathrm{x}} ; 1-k_{\mathrm{N}}, 1,1,1\right)$ \\
functions & $\mu_{\mathrm{SP}}\left(k_{\mathrm{x}}\right)=\operatorname{trapezoid}\left(k_{\mathrm{x}} ; 1,1,1,1+k_{\mathrm{P}}\right)$ \\
& $\mu_{\mathrm{LP}}\left(k_{\mathrm{x}}\right)=\operatorname{trapezoid}\left(k_{\mathrm{x}} ; 1,1+k_{\mathrm{P}}, \infty, \infty\right)$ \\
\hline
\end{tabular}




\begin{tabular}{|l|l|}
\hline Rule base & $\begin{array}{l}\text { IF } k_{\mathrm{x}}=\text { LN THEN } y\left(x, x_{\mathrm{m}}\right)=f_{\mathrm{LN}} \\
\text { IF } k_{\mathrm{x}}=\text { SN THEN } y\left(x, x_{\mathrm{m}}\right)=f_{\mathrm{SN}} \\
\text { IF } k_{\mathrm{x}}=\text { SP THEN } y\left(x, x_{\mathrm{m}}\right)=f_{\mathrm{SP}} \\
\text { IF } k_{\mathrm{x}}=\text { LP THEN } y\left(x, x_{\mathrm{m}}\right)=f_{\mathrm{LP}}\end{array}$ \\
\hline $\begin{array}{l}\text { Conclusion } \\
\text { functions }\end{array}$ & $f_{L N}=x$ \\
$f_{S N}=\frac{1}{2} \frac{\left(k_{P}+2 k_{N}\right) x+\left(k_{P}-k_{P} k_{N}\right) x_{m}}{k_{N}+k_{P}}$ \\
$f_{S P}=\frac{1}{2} \frac{\left(2 k_{P}+k_{N}-k_{P} k_{N}\right) x_{m}+k_{N} x}{k_{N}+k_{P}}$ \\
$f_{L P}=x_{m}$
\end{tabular}

The Figure 5 shows that the resulting approximation $y\left(x, x_{\mathrm{m}}\right)$ of $\min \left(x, x_{\mathrm{m}}\right)$ is smooth, free of overshoots but not symmetric.
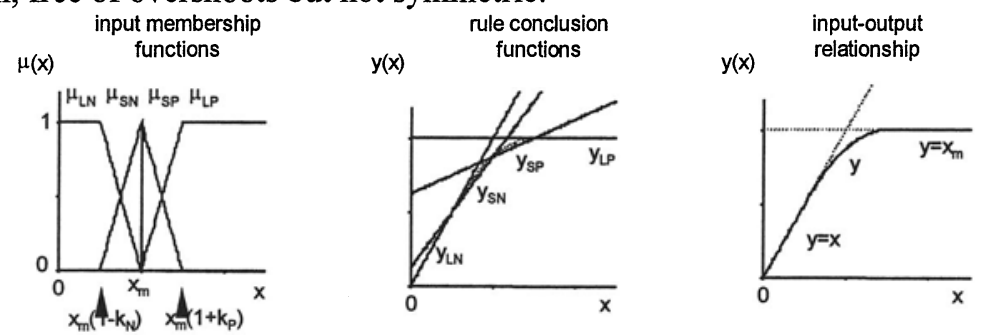

Figure 5. System with 4 Rules: input membership functions, conclusion functions and input-output relationship.
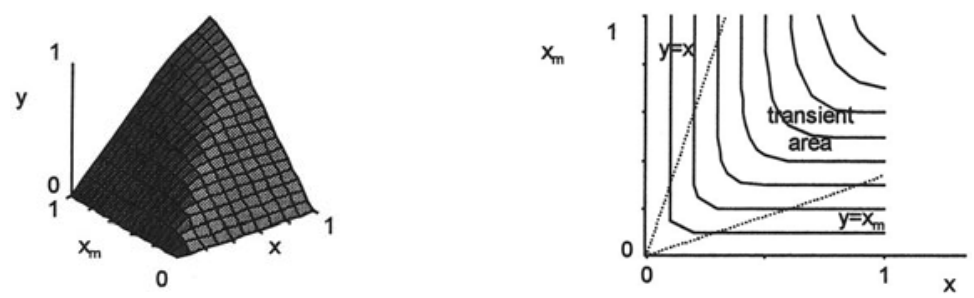

Figure 6. Fuzzy approximation of $\min \left(x, x_{\mathrm{m}}\right)$ by a fuzzy system with 4 Rules.

\section{APPLICATIONS}

\subsection{Building blocks}

To make the controller design easier, building blocks are used to encapsulate the bare function $\min \left(x, x_{\mathrm{m}}\right)$. Typical blocks are the 1 quadrant limiter and the 2 quadrant limiter (performs the limitation with respect to both $x_{\mathrm{m}}$ and $-x_{\mathrm{m}}$ ). 


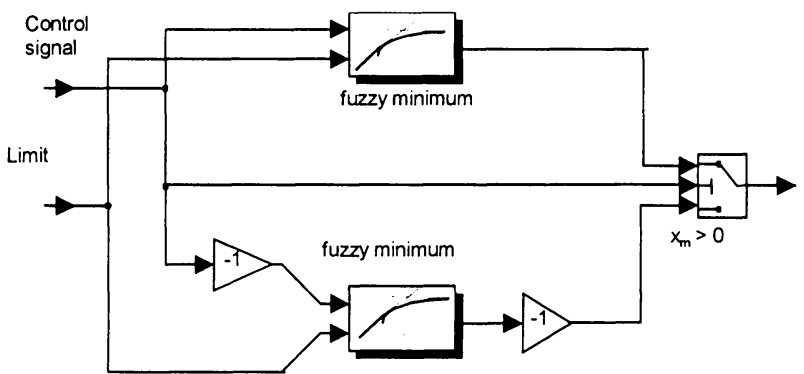

Figure 7. Block structure of the 2 quadrant limter.

\subsection{Desired position trajectory with s-shaped velocity profile}

Typical desired trajectory in control applications is composed of several transitions between specific setpoints (desired positions) which: (a) are continuous up to the $2^{-}$ nd derivative, (b) do not exceed velocity and acceleration limits, (c) reach the final position $x_{\mathrm{f}}$ in minimum time.

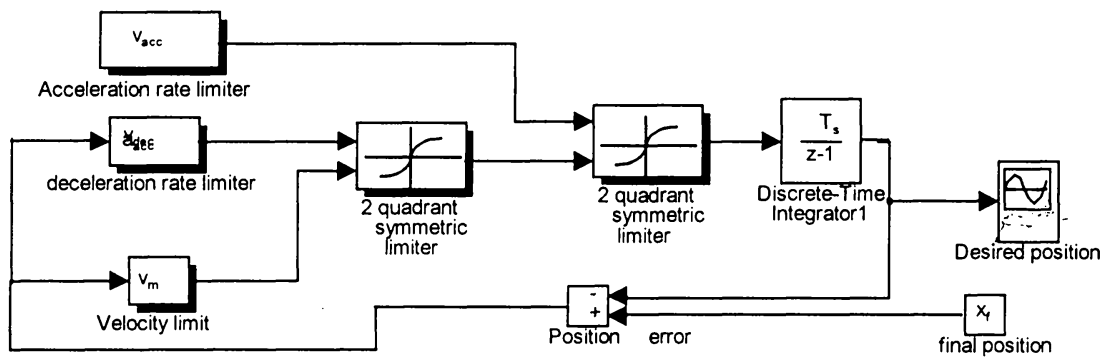

Figure 8. Block structure of the generator of desired trajectory with constant final position and s-shaped velocity profile.
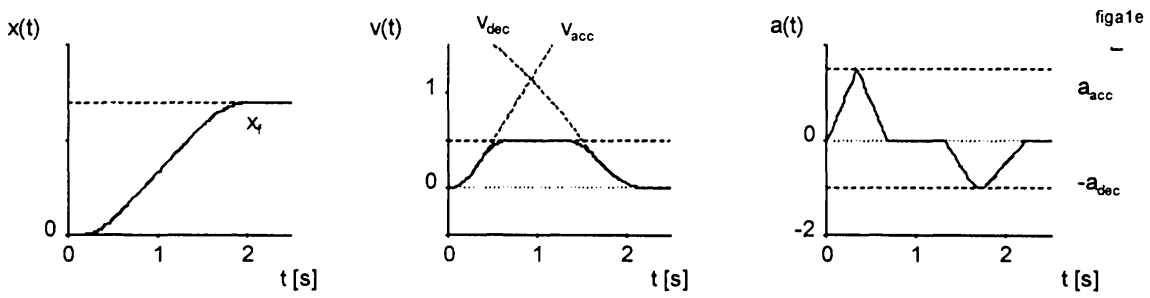

Figure 9. The full desired trajectory with s-shaped velocity profile generated according to the block diagram in Figure 8. 


\subsection{Drive Control}

We want to operate drives close to their limits with minimum or zero margins in control actions. Therefore, we use two dedicated controllers and blend their signals using the 2-quadrant minimum.

Combination of PID position control and force control is used as an example of such blending. The extrusion of the plastic material in moulding machines can be used as a typical example of this type of control.
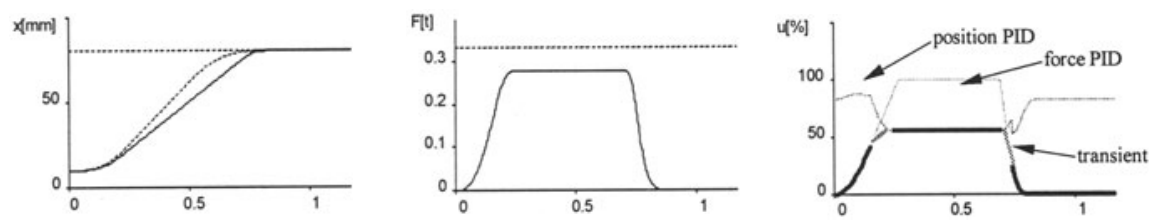

Figure 10. Position control of a drive with force limitation.

\section{CONCLUSIONS}

Properties of different methods of approximation of the function $\min \left(x, x_{\mathrm{m}}\right)$ are summarised in the Table 1 . The non-symmetric fuzzy algorithm with 4 rules proved to be the best candidate in control applications because it needs the lowest number of calculations.

The fuzzy approximation was used in 2 practical control problems: the generation of desired trajectory with s-shaped velocity profile and in the combination of outputs of two and more dedicated controllers.

Table 1. Properties of different methods

\begin{tabular}{lccccccc}
\hline Method & $\begin{array}{c}\text { Degree of } \\
\text { continuity }\end{array}$ & $\begin{array}{c}\text { Symme } \\
\text { tric }\end{array}$ & $\begin{array}{c}\text { Over } \\
\text { shoot }\end{array}$ & \multicolumn{4}{c}{ Number of operations } \\
& 0 & yes & no & - & - & - & 1 \\
\hline Minimum & 0 & yes & no & 3 & - & - & 1 \\
Power N & $\mathrm{n}$ & no & yes & - & - & 4 & 4 \\
Fuzzy 2 rules & 0 & no & no & - & - & 4 & 4 \\
Fuzzy 4 rules & 1 & & & & & \\
\hline
\end{tabular}

\section{BIBLIOGRAPHY}

[1] Horáček, P. Binder, Z.: Nonlinear Control of Systems with Hydraulic Cylinders. In: Proceedings of International Workshop on Applied Automatic Control WAAC'93, Prague. 1993, pp 73-76.

[2] Sládek,B.: Fuzzy approximation of functions minimum and maximum. Internal report No: GL-51/97, CTU Prague 